\title{
A patch that fixes insulin delivery
}

Type 1 diabetes (T1D) is an autoimmune disorder that currently affects more than 1 million people in the US and more than 370 million people worldwide. In T1D, the immune system mistakenly identifies pancreatic beta cells as foreign material and attacks and destroys them. Beta cells are the body's natural producers of insulin, which helps to process glucose into energy. Without enough insulin, glucose accumulates in the blood, which can have negative effects on the body. People with T1D must therefore monitor their blood glucose level closely and administer insulin when needed to maintain normoglycemia (blood glucose level within the normal range). Many patients monitor themselves by pricking a finger to obtain a drop of blood for glucose testing and administer insulin as needed via subcutaneous injection using a syringe. This process must be repeated several times per day, and it can be demanding, painful, imprecise and less than $100 \%$ effective at achieving normoglycemia. Closed-loop systems that involve direct connections between sensors that track blood glucose and pumps that administer insulin can improve the precision of glycemic control

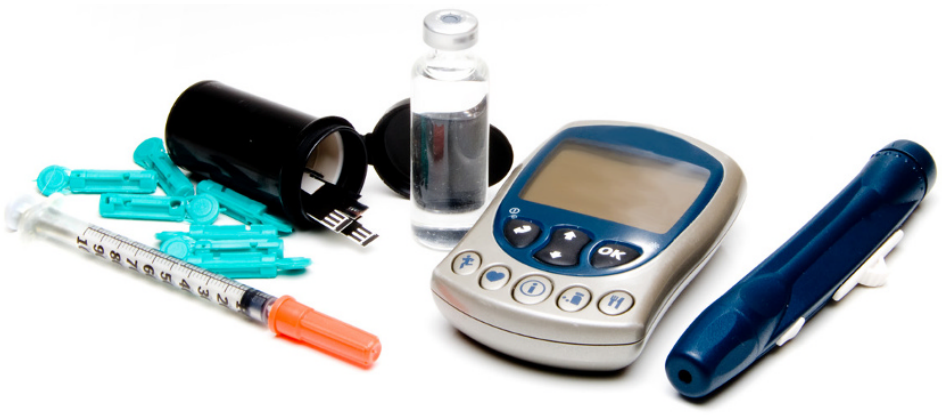

but can still be painful and demanding to use. Therefore, there is a great need for better glucose-responsive insulin delivery systems that are fast-acting, easy to use and biocompatible.

One approach to developing such a system is to mimic the natural function of beta cells, which store insulin in tiny sacs called vesicles and release it into the bloodstream when they sense increases in blood glucose. Recent progress on this front comes from scientists in the Joint Department of Biomedical Engineering at University of North Carolina at Chapel Hill and North Carolina State University (Raleigh). Led by $\mathrm{Zhen} \mathrm{Gu}$, the team created synthetic vesicles that release insulin in response to a high glucose level and formulated the vesicles into a small patch that can be applied painlessly to the skin. "We have designed a patch for diabetes that works fast, is easy to use, and is made from nontoxic, biocompatible materials," said Gu in a press release.

In a mouse model of T1D, use of the patch lowered blood glucose level from $>500 \mathrm{mg} /$ $\mathrm{dl}$ to $\sim 200 \mathrm{mg} / \mathrm{dl}$ within about $30 \mathrm{~min}$ and maintained normoglycemia $(<200 \mathrm{mg} / \mathrm{dl}$ glucose) for up to $4 \mathrm{~h}$ (Proc. Natl. Acad. Sci. USA 112, 8260-8265; 2015). “This smart insulin patch... offers a clinical opportunity for closed-loop delivery of insulin in a fast glucose-responsive, pain-free, and safe manner," the authors wrote.

Monica Harrington

\section{CHRONIC PATN: IT'S DIFFERENT FOR GIRIS}

The study of pain is essential to biomedical research for many reasons. Signs and reports of pain can inform diagnoses and prognoses by conveying the severity of symptoms or the efficacy of treatment. Untreated pain can also have a deleterious effect on a patient's wellbeing and quality of life. Finally, in biological research it is the ethical responsibility of investigators to reduce and minimize pain in their subjects whenever possible. It is important, therefore, to understand the physiology of pain and the mechanisms by which treatments can relieve pain in humans and animals.

Previous research on the pathophysiology of pain has yielded one prevailing model of chronic pain, wherein glial cells are thought to initiate and maintain chronic pain in response to peripheral injuries. Recent findings indicate that this explanation is only half-true: in women, chronic pain is instead mediated by T cells (Nat. Neurosci. doi:10.1038/nn.4053; published online 29 June 2015).

"For the past 15 years scientists have thought that microglia controlled the volume knob on pain, but this conclusion was based on research using almost exclusively male mice," said Jeffrey Mogil of McGill University (Montreal, Canada), who led the study alongside colleagues from the US and Canada.

Mogil's team discovered this difference when they used the spared nerve injury technique to model persistent neuropathic pain in both male and female mice. All mice showed allodynia, or mechanical hypersensitivity, after the injury, but only male mice lost their allodynia when injected with glial inhibitors. Females continued to show allodynia after receiving the same injection and after receiving an injection of saporin toxin that depleted their microglia.

The researchers then recreated the chronic pain model using strains of mice that are deficient in T cells and found that glial inhibitors successfully relieved allodynia in both males and females. Taken together, these findings suggest that the female pain response induces allodynia preferentially through $\mathrm{T}$ cells, as seen in the wild-type mice, but functions through glial cells only when T cells are deficient.

The presence of a sexual dimorphism at the physiological level is not surprising in and of itself. Rather, the authors note, it is surprising that this difference has remained unnoticed for so long. These findings advance our understanding of pain in mice and potentially in humans, but moreover they underscore the importance of reconsidering accepted conventions and assumptions of common research models.

Gregory D. Larsen 